\title{
Antituberculosis BCG vaccination: more reasons for varying innate and adaptive immune responses
}

\author{
S. Prentice ${ }^{1}$ and H.M. Dockrell ${ }^{2}$ \\ 'Department of Paediatrics, East and North Hertfordshire NHS Trust, Stevenage, United Kingdom. ${ }^{2}$ Department of Infection Biology, London School of Hygiene and Tropical Medicine, \\ London, United Kingdom.
}

Bacillus Calmette-Guérin (BCC) vaccination induces variable protection against pulmonary tuberculosis (TB), and a more effective TB vaccine is needed. The potential for BCG to provide protection against heterologous infections, by induction of innate immune memory, is increasingly recognized. These nonspecific responses may substantially benefit public health, but are also variable. In this issue of the $J C l$, Koeken and de Bree et al. report that BCC reduces circulating inflammatory markers in males but not in females, while de Bree and Mouritis et al. describe how diurnal rhythms affect the degree of BCG-induced innate memory. These studies further delineate factors that influence the magnitude of responses to BCC and may be crucial to harnessing its potential benefits.

\section{A long history}

Bacillus Calmette-Guérin (BCG) is the oldest vaccine in use today. Yet, despite widespread use for nearly 100 years, much about it remains to be discovered. Epidemiological studies have shown that BCG can provide protection against the disseminated forms of tuberculosis (TB) in young children, but it induces very variable protection against pulmonary $\mathrm{TB}$ in adolescents and adults (1). Factors such as geographical location, age at vaccination, and vaccine strain have all been associated with varying BCG-specific responses, but they do not completely explain the heterogeneity seen $(1,2)$. The variable efficacy of BCG against TB has driven the search for an improved, more efficacious TB vaccine (3). However, as some of the current vaccine candidates would be given as a boosting vaccine following BCG, and others are genetically modified live BCG vaccines (or other live mycobacteria), we still need to understand why BCG induces such variable protection. Proven correlates of protection against TB also remain elu- sive, despite a resurgence in mechanistic research on BCG. Heterogeneity in an individual's immune response to $\mathrm{BCG}$ vaccination is likely to contribute considerably to the challenges in identifying specific correlates of protection.

\section{Increased resistance to bacterial and viral infections?}

More recently, the possibility that BCG may produce protection against heterologous, nontuberculous, infectious disease has been recognized. Trials have shown that BCG has protective effects against morbidity and mortality from unrelated infections in neonates and young children, in countries with high infectious disease burden (4, 5), corroborating epidemiological studies associating BCG with reductions in sepsis and lower respiratory tract infections in children $(6,7)$. Mechanistic studies suggest that this heterologous protection afforded by BCG may result, at least in part, from the induction of nonspecific innate immune memory via epigenetic reprogramming of myeloid cells (8). Heterogeneity in the non-

Related Articles: pp. 5591 and 5603

Conflict of interest: HMD and SP declare that no conflict of interest exists.

specific beneficial effects induced by BCG has also been noted (9).

Interest in BCG has markedly increased since the start of the COVID-19 pandemic, with the possibility that BCG might provide protection against SARSCoV-2 infection or disease, in the absence of a specific vaccination, being explored. Given that BCG protects against viral infections in a number of animal models (10) and has been shown to protect against viremia after experimental infection with the attenuated yellow fever vaccine (11), hopes are high. Trials are ongoing to test whether BCG vaccination would prevent SARS2-CoV-2 infection or severe COVID-19 disease in healthcare workers and the elderly (12).

\section{Host and environmental factors that impact immune response}

In this climate, the 2 papers by Koeken and de Bree et al. (13) and de Bree and Mouritis et al. (14) in this issue of the JCI provide further important insights into host and environmental factors that may modulate immune responses, and in particular innate immunity, following BCG vaccination. The study by Koeken and de Bree et al. (13) investigated how the Bulgarian strain of the BCG vaccine regulated markers of inflammation in a cohort of 303 healthy volunteers recruited in The Netherlands, and how sex affected these responses. Twenty-five circulating inflammatory markers were reduced 2 weeks after BCG vaccination; possibly more interestingly, 10 markers remained lower 3 months after vaccination. Further, individuals with larger BCG scars showed a greater decrease in some inflammatory markers after BCG vaccination. Strikingly, males had higher prevaccination concentrations of 41 of the inflammatory markers, with significant decreases after vaccination, while BCG had no significant effects on the concentrations of these inflammatory mediators in 
females after adjusting for multiple testing (13). Reduced expression of 4 inflammatory markers (including TNF ligand superfamily member 12 [TWEAK] and sirtuin 2 [SIRT2]) persisted 3 months after BCG, a finding that was validated in 39 additional individuals from 3 other studies (13).

The finding of reduced systemic inflammation induced by BCG in the study by Koeken and de Bree et al. (13) is striking in the context of the potential use of BCG for prevention of COVID-19 infection/disease, as severe forms of COVID-19 are characterized by a hyperinflammatory state. Dexamethasone, a corticosteroid with potent antiinflammatory properties, is the only current treatment with proven benefits for SARS-CoV-2 (15). The Koeken and de Bree study (13), therefore, provides further evidence to suggest that the nonspecific effects of BCG might theoretically be harnessed in the fight against COVID-19.

The finding by Koeken and de Bree et al. (13) of sex-differential effects of BCG on systemic inflammation is also important. Earlier trials in Guinea-Bissau, where BCG was given to low-birth weight infants, showed greater nonspecific benefits in males compared with females (4), and a similar effect has been observed in East Africa (5). A metaanalysis of more than 3,000 COVID-19 patients in China showed that males had a higher rate of disease progression than females (16), so a more marked effect of BCG on inflammation might substantially benefit males. Sex differences are being increasingly recognized throughout medicine, with impacts on drug efficacy and side effects, immunity, vaccine responses, and the presentation, course, and outcomes of many diseases (17). It is imperative, therefore, that going forward, human studies, including vaccination trials, should recruit a balanced number of male and female participants, where appropriate, and report sex-disaggregated results as standard.

Another risk factor for severe COVID-19 disease is diabetes, which also induces changes in inflammation. A recent study showed how the interaction of infection with TB and type 2 diabetes, or even intermediate hyperglycemia insufficient to diagnose diabetes, resulted in marked increases in the expression of markers of inflammation while dampening the interferon response (18). Potentially, diabetes could exacerbate COVID-19 disease via a similar mechanism, so reducing inflammation via BCG vaccination would be beneficial.

The study by de Bree and Mouritis et al. (14) shows that the circadian rhythm can also influence the immunogenicity and immune modulation induced by BCG vaccination; trained immunity was greater when BCG vaccination was performed at 8 to 9 am rather than $6 \mathrm{pm}$. Again, there is a large body of literature on how peripheral blood counts and immune parameters are influenced by the time of day (19). Studies in mice have also shown circadian variation in LPS-induced secretion of TNF- $\alpha$ and IL-6, with the greatest cytokine secretion occurring in daytime (20), as well as a higher mortality rate in mice administered with high doses of LPS at the end of the day than those administered in the middle of the night (21). Vaccination in the morning has also been shown to increase antibody responses (22). Diurnal rhythms link to metabolic processes and immune function (23) and metabolism plays a key role in training and the induction of nonspecific immunity by BCG (24). More research will be needed to determine the importance of circadian rhythms on vaccine responses in neonates, as the circadian system is immature at birth (25), and in the elderly, as disordered circadian rhythms can occur with age (26).

\section{Conclusions and future studies}

Overall, these studies put 2 more pieces into the jigsaw puzzle that, when complete, will show us why BCG does what it does in different circumstances and different individuals in the real world, and how to ensure that optimal responses are induced. Other pieces of the puzzle remain incomplete. Factors including heterogeneity in the BCG vaccine itself, vaccination history with other vaccines, maternal factors, nutrition, our microbiota, prior and subsequent infections with other mycobacteria and unrelated viruses, as well as genetics may also be important $(9,27,28)$. With the advent of big data and artificial intelligence, we should be better able to integrate the large data sets that these types of studies produce and better understand the complex and varied immune responses seen in human populations.
Complete identification of the causes of heterogeneity in vaccine responses may help in the search for correlates of specific and nonspecific BCG-induced protection. In turn, robust correlates of protection should help us to investigate which of the various factors influencing BCG responses are relevant on a public health scale. Immunization schedules are already complex. Are the enhanced individual responses provided by early morning BCG sufficiently profound to limit vaccinations to the morning, potentially at the expense of broad vaccine coverage? If the sexdifferential effects of BCG are confirmed to be clinically important, would a different BCG vaccination dose or schedule in girls and boys be acceptable to the general public? In this era of increasing vaccine hesitancy, it is imperative that questions such as these are answered.

In the meantime, we can only hope that BCG will provide some useful protection against SARS2-CoV-2 while we wait for a specific vaccine. Based on the findings of Koeken and de Bree et al. (13) and de Bree and Mouritis et al. (14), prudent clinical trials would assess the efficacy of BCG for protection against COVID-19 following morning vaccination. If protection is induced, it will be interesting to know whether these beneficial effects are present to an equal extent in males and females.

\section{Acknowledgments}

HMD and SP thank F.J. Sanchez-Garcia and Jackie Cliff for helpful discussions and for reviewing the manuscript. F.J. Sanchez-Garcia, S. Smith, and HMD are recipients of a grant from the GCRF Networks in Vaccines Research and Development VALIDATE Network (grant MR/ R00585011, P020).

Address correspondence to: H.M. Dockrell, Department of Infection Biology, London School of Hygiene and Tropical Medicine, Keppel Street, London WC1E 7HT, United Kingdom. Phone: 0044.207.927.2466; Email: hazel.dockrell @lshtm.ac.uk.

\footnotetext{
1. Mangtani P, et al. Protection by BCG vaccine against tuberculosis: a systematic review of randomized controlled trials. Clin Infect Dis. 2014;58(4):470-480.

2. Grassly NC, Kang G, Kampmann B. Biological
} challenges to effective vaccines in the devel- 
oping world. Philos Trans R Soc Lond B Biol Sci. 2015;370(1671):20140138.

3. Brazier B, McShane H. Towards new TB vaccines. Semin Immunopathol. 2020;42(3):315-331.

4. Biering-Sørensen S, Jensen KJ, Monterio I, Ravn $\mathrm{H}$, Aaby P, Benn CS. Rapid protective effects of early BCG on neonatal mortality among low birth weight boys: observations from randomized trials. J Infect Dis. 2018;217(5):759-766.

5. Prentice S, et al. BCG-induced non-specific protection against heterologous infectious disease in Ugandan neonates: an investigator-blind randomised controlled trial. Lancet Infect Dis. In press.

6. de Castro MJ, Pardo-Seco J, Martinón-Torres F. Nonspecific (heterologous) protection of neonatal BCG vaccination against hospitalization due to respiratory infection and sepsis. Clin Infect Dis. 2015;60(11):1611-1619.

7. Hollm-Delgado M, Stuart EA, Black RE. Acute lower respiratory infection among bacille Calmette-Guérin (BCG)-vaccinated children. Pediatrics. 2014;133(1):e73-81.

8. Netea MG, et al. Trained immunity: A program of innate immune memory in health and disease. Science. 2016;352(6284):aaf1098.

9. Angelidou A, et al. BCG as a case study for precision vaccine development: lessons from vaccine heterogeneity, trained immunity, and immune ontogeny. Front Microbiol. 2020;11:332.

10. Moorlag SJCFM, Arts RJW, van Crevel R, Netea MG. Non-specific effects of BCG vaccine on viral infections. Clin Microbiol Infect. 2019;25(12):1473-1478.

11. Arts RJW, et al. BCG vaccination protects against experimental viral infection in humans through the induction of cytokines associated with trained immunity. Cell Host Microbe. 2018;23(1):89-100.e5.

12. Curtis N, Sparrow A, Ghebreyesus TA, Netea MG. Considering BCG vaccination to reduce the impact of COVID-19. Lancet. 2020;395(10236):1545-1546.

13. Koeken VACM, et al. BCG vaccination in humans inhibits systemic inflammation in a sex-dependent manner. JClin Invest. 2020;130(10):5591-5602.

14. de Bree LCJ, et al. Circadian rhythm influences induction of trained immunity by BCG vaccination. J Clin Invest. 2020;130(10):5603-5617.

15. RECOVERY Collaborative Group, et al. Dexamethasone in hospitalized patients with COVID-19 - preliminary report [published online July 17, 2020]. N Engl JMed. https://doi. org/10.1056/NEJMoa2021436.

16. Zheng Z, et al. Risk factors of critical \& mortal COVID-19 cases: A systematic literature review and meta-analysis. J Infect. 2020;81(2):e16-e25.

17. Klein SL, Flanagan KL. Sex differences in immune responses. Nat Rev Immunol. 2016;16(10):626-638.

18. Eckold C, et al. Impact of intermediate hyperglycaemia as well as diabetes on immune dysfunction in tuberculosis [published online June 13, 2020]. Clin Infect Dis. https://doi.org/10.1093/ cid/ciaa751.

19. Scheiermann C, Gibbs J, Ince L, Loudon A. Clocking in to immunity. Nat Rev Immunol. 2018;18(7):423-437.
20. Keller M, et al. A circadian clock in macrophages controls inflammatory immune responses. Proc Natl Acad Sci U S A. 2009;106(50):21407-21412.

21. Marpegan L, Leone MJ, Katz ME, Sobrero PM, Bekinstein TA, Golombek DA. Diurnal variation in endotoxin-induced mortality in mice: correlation with proinflammatory factors. Chronobiol Int. 2009;26(7):1430-1442.

22. Long JE, Drayson MT, Taylor AE, Toellner KM, Lord JM, Phillips AC. Morning vaccination enhances antibody response over afternoon vaccination: A cluster-randomised trial. Vaccine. 2016;34(24):2679-2685.

23. Aguilar-López BA, Moreno-Altamirano MMB, Dockrell HM, Duchen MR, Sánchez-García FJ. Mitochondria: an integrative hub coordinating circadian rhythms, metabolism, the microbiome, and immunity. Front Cell Dev Biol. 2020;8:51.

24. Arts RJW, et al. Immunometabolic pathways in BCG-induced trained immunity. Cell Rep. 2016;17(10):2562-2571.

25. Rivkees SA. Emergence and influences of circadian rhythmicity in infants. Clin Perinatol. 2004;31(2):217-228.

26. Cornelissen G, Otsuka K. Chronobiology of aging: a mini-review. Gerontology. 2017;63(2):118-128.

27. Butkeviciute E, Jones CE, Smith SG. Heterologous effects of infant BCG vaccination: potential mechanisms of immunity. Future Microbiol. 2018;13:1193-1208.

28. Zimmermann P, Curtis N. Factors that influence the immune response to vaccination. Clin Microbiol Rev. 2019;32(2):e00084-18. 